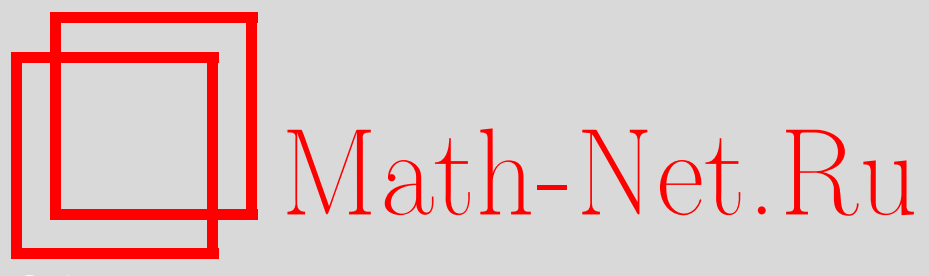

С. М. Рацеев, Функции сложности многообразий алгебр Лейбница с нильпотентным коммутантом, Матем. заметки, 2015, том 98, выпуск 3, 472-475

DOI: https://doi.org/10.4213/mzm10819

Использование Общероссийского математического портала Math-Net.Ru подразумевает, что вы прочитали и согласны с пользовательским соглашением http://www . mathnet.ru/rus/agreement

Параметры загрузки:

IP: 3.85 .7 .115

26 апреля 2023 г., 15:05:48

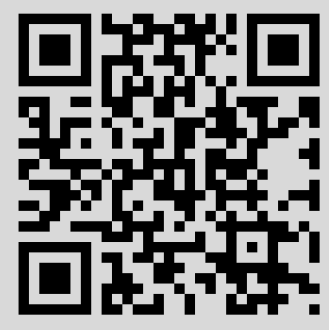




\section{Функции сложности многообразий алгебр Лейбница с нильпотентным коммутантом}

\section{С. М. Рацеев}

Алгебры Лейбница определяются тождеством $(x y) z=(x z) y+x(y z)$ и являются обобщениями алгебр Ли. Данные алгебры начали активно изучаться в 90-х годах, начиная с работы Лодея [1]. Алгебры Лейбница связаны естественным образом с дифференциальной геометрией, гомологической алгеброй, классической алгебраической топологией и некоммутативной геометрией [1].

Пусть $L(X)$ - свободная алгебра Лейбница над полем $K$, где $X=\left\{x_{1}, x_{2}, \ldots\right\}$ - счетное множество свободных образующих, $P_{n}$ - подпространство в $L(X)$, состоящее из всех полилинейных элементов степени $n$ от переменных $x_{1}, \ldots, x_{n}$. Пусть $\mathbf{V}$ - многообразие алгебр Лейбница, $\operatorname{Id}(\mathbf{V})$ - идеал тождеств многообразия $\mathbf{V}$ в свободной алгебре $L(X)$ (все необходимые определения и сведения из теории PI-алгебр можно найти например в монографии [2]). Обозначим

$$
P_{n}(\mathbf{V})=P_{n} /\left(P_{n} \cap \operatorname{Id}(\mathbf{V})\right), \quad c_{n}(\mathbf{V})=\operatorname{dim} P_{n}(\mathbf{V}) .
$$

Будем опускать скобки при их левонормированной расстановке: $((a b) c)=a b c$.

В следующей лемме, которую нетрудно проверить, приводится конструкция алгебр Лейбница на основе ассоциативных алгебр.

Лемма 1. Пусть $A$ - некоторая ассоииативная алгебра с операиией умножения $\wedge$ над произвольным полем $K$. На декартовом квадрате $B=A \times A$ определим операчии сложения и умножения элементов множества $B$, а также операчию умножения на элементы поля $K$ :

$$
\begin{aligned}
\left(x_{1}, x_{2}\right)+\left(y_{1}, y_{2}\right) & =\left(x_{1}+y_{1}, x_{2}+y_{2}\right), \\
\left(x_{1}, x_{2}\right)\left(y_{1}, y_{2}\right) & =\left(\left[x_{1}, y_{1}\right],\left[x_{2}, y_{1}\right]\right), \\
\alpha\left(x_{1}, x_{2}\right) & =\left(\alpha x_{1}, \alpha x_{2}\right),
\end{aligned}
$$

где $[x, y]=x \wedge y-y \wedge x, \alpha \in K,\left(x_{1}, x_{2}\right),\left(y_{1}, y_{2}\right) \in B$. Тогда полученная алгебра $B$ будет являться алгеброй Лейбница.

Определим многообразие алгебр Лейбница $\widetilde{\mathbf{N}_{s} \mathbf{A}}$ тождеством

$$
\left(x_{1} x_{2}\right) \ldots\left(x_{2 s+1} x_{2 s+2}\right)=0 .
$$

В работах [3], [4] приводятся оценки роста подмногообразий в $\widetilde{\mathbf{N}_{s} \mathbf{A}}$, а в работе [5] получены эквивалентные условия для значений экспонент многообразий алгебр Лейбница с нильпотентным коммутантом.

Пусть $U T_{s}=U T_{s}(K)$ - алгебра верхнетреугольных матриц порядка $s$ над полем $K, \widetilde{U}_{s}=$ $U T_{s} \times U T_{s}-$ алгебра Лейбница, построенная с помощью леммы 1 . Нетрудно видеть, что $\widetilde{U}_{s} \in \widehat{\mathbf{N}_{s-1} \mathbf{A}}$.

Теорема 1. (i) В случае произвольного поля $K$ следующие полилинейные элементы от переменных $x_{1}, \ldots, x_{n}$ образуют базис пространства $P_{n}\left(\widetilde{U}_{s}\right), s \geqslant 2$ :

$$
\begin{gathered}
x_{m_{1}} x_{m_{2}} x_{i_{1}} \ldots x_{i_{k}}\left(x_{11} \ldots x_{1 a_{1}}\right) \ldots\left(x_{c 1} \ldots x_{c a_{c}}\right) \\
m_{1}, m_{2} \in\{1,2, \ldots n\}, \quad m_{1} \neq m_{2}, \quad k \geqslant 0, \quad i_{1}<\ldots<i_{k}, \quad a_{1}, \ldots, a_{c} \geqslant 2, \quad 0 \leqslant c \leqslant s-2,
\end{gathered}
$$

DOI: $10.4213 / \mathrm{mzm} 10819$ 
и переменные в каждой скобке $\left(x_{j 1} \ldots x_{j a_{j}}\right)$ упорядочены следующим образом:

$$
j 1>j 2<\cdots<j a_{j} .
$$

(ii) Если характеристика поля $K$ равна нулю, то тождество

$$
\left(x_{1} x_{2}\right) \ldots\left(x_{2 s-1} x_{2 s}\right)=0
$$

порождает идеал тождеств алгебры $\widetilde{U}_{s}$ (иными словами, алгебра $\widetilde{U}_{s}$ является носителем многообразия $\widetilde{\mathbf{N}_{s-1} \mathbf{A}}$ ).

ДоказАтельство. Пусть $L=L(X)$ - свободная алгебра Лейбница. Рассмотрим следующие векторные пространства:

$$
R_{c, n}=P_{n}\left(\left(L^{2}\right)^{c+1} /\left(L^{2}\right)^{c+2}\right), \quad c=0,1, \ldots .
$$

Заметим, что $P_{n}\left(\widetilde{\mathbf{N}_{s} \mathbf{A}}\right) \cong \bigoplus_{c=0}^{s-1} R_{c, n}$. Пространство $R_{c, n}$ представимо в виде линейной оболочки следующих полилинейных элементов от переменных $x_{1}, \ldots, x_{n}$ :

$$
\begin{array}{r}
R_{c, n}=\left\langle x_{m_{1}} x_{m_{2}} x_{i_{1}} \ldots x_{i_{k}}\left(x_{11} \ldots x_{1 a_{1}}\right)\left(x_{21} \ldots x_{2 a_{2}}\right) \ldots\left(x_{c 1} \ldots x_{c a_{c}}\right)\right. \\
\left|k \geqslant 0, a_{i} \geqslant 2, i=1, \ldots, c, a_{1}+\cdots+a_{c}+k+2=n\right\rangle_{K} .
\end{array}
$$

В элементах пространства $R_{c, n}$ можно менять местами переменные $x_{i_{1}} \ldots x_{i_{k}}$, а также переменные в любой $j$-й скобке, $j=1, \ldots, c$, начиная с 3 -й позиции, поскольку, меняя местами две рядом стоящие переменные, мы дополнительно получаем элемент из $\left(L^{2}\right)^{c+2}$. Также используя тождество $x(y y)=0$, которое выполнено в любой алгебре Лейбница, можно в элементах пространства $R_{c, n}$ в любой $j$-й скобке, $j=1, \ldots, c$, переменные упорядочить так, чтобы выполнялись неравенства $j 1>j 2<\cdots<j a_{j}$. Так как $\widetilde{U}_{s} \in \widetilde{\mathbf{N}_{s-1} \mathbf{A}}$, то $P_{n}\left(\widetilde{U}_{s}\right)$ является линейной оболочкой элементов вида (1) с условиями (2).

Каждому элементу $\omega$ вида (1) сопоставим такой упорядоченный набор чисел (длины скобок): $I(\omega)=\left(k+2, a_{1}, \ldots, a_{c}\right)$. На множестве элементов данного вида определим частичную упорядоченность. Пусть $\omega_{1}, \omega_{2}$ - элементы вида (1). Определим $\omega_{1} \prec \omega_{2}$, если набор $I\left(\omega_{1}\right)$ лексикографически слева направо меньше набора $I\left(\omega_{2}\right)$.

Предположим, что для некоторого $n$ элементы вида (1) с условиями (2) линейно зависимы в $P_{n}\left(\widetilde{U}_{s}\right)$. В полученной нетривиальной линейной комбинации элементов вида (1) зафиксируем такое слагаемое, которое имеет ненулевой коэффициент, минимальное значение $c$ и является минимальным элементом относительно порядка $\prec$ среди всех элементов с ненулевыми коэффициентами и минимальными значениями $c$. Пусть это слагаемое имеет вид

$$
\alpha x_{m_{1}} x_{m_{2}} x_{i_{1}} \ldots x_{i_{k}}\left(x_{11} \ldots x_{1 a_{1}}\right) \ldots\left(x_{c 1} \ldots x_{c a_{c}}\right), \quad 0 \neq \alpha \in K .
$$

Во всей линейной комбинации сделаем такую подстановку:

$$
\begin{aligned}
& x_{m_{1}} \rightarrow\left(0, e_{11}\right), \quad x_{m_{2}} \rightarrow\left(e_{12}, 0\right), \quad x_{i_{1}} \rightarrow\left(e_{22}, 0\right), \quad x_{i_{2}} \rightarrow\left(e_{22}, 0\right), \quad \ldots, \quad x_{i_{k}} \rightarrow\left(e_{22}, 0\right), \\
& x_{11} \rightarrow\left(e_{23}, 0\right), \quad x_{12} \rightarrow\left(e_{33}, 0\right), \quad x_{13} \rightarrow\left(e_{33}, 0\right), \quad \ldots, \quad x_{1 a_{1}} \rightarrow\left(e_{33}, 0\right), \\
& x_{21} \rightarrow\left(e_{34}, 0\right), \quad x_{22} \rightarrow\left(e_{44}, 0\right), \quad x_{23} \rightarrow\left(e_{44}, 0\right), \quad \ldots, \quad x_{2 a_{2}} \rightarrow\left(e_{44}, 0\right), \\
& x_{c 1} \rightarrow\left(e_{c+1, c+2}, 0\right), \quad x_{c 2} \rightarrow\left(e_{c+2, c+2}, 0\right), \quad x_{c 3} \rightarrow\left(e_{c+2, c+2}, 0\right), \quad \ldots, \quad x_{c a_{c}} \rightarrow\left(e_{c+2, c+2}, 0\right),
\end{aligned}
$$

где $e_{i j}$ - матричные единички. Тогда все слагаемые, кроме рассматриваемого, будут равны нулю, а данное слагаемое будет равно $\alpha\left(0, e_{1, c+2}\right)$. Понятно, что равенство $\alpha\left(0, e_{1, c+2}\right)=$ $(0,0)$ выполнено лишь в случае $\alpha=0$. Противоречие. 
Ю. П. Размыслов предложил рассматривать для произвольного многообразия $\mathbf{V} ф y н к-$ цию сложности

$$
\mathscr{C}(\mathbf{V}, z)=\sum_{n=1}^{\infty} \frac{c_{n}(\mathbf{V})}{n !} z^{n}, \quad z \in \mathbb{C} .
$$

Это один из примеров экспоненциальной производящей функции [6]. Функции сложности оказываются полезными для вычисления асимптотики роста многообразий. Применение функций сложности для многообразий алгебр Ли оказалось плодотворным и привело к классификации типов роста [7], [8].

Рассмотрим функцию сложности в более общем виде. Пусть имеется алгебра (группоид), порожденная счетным множеством $X=\left\{x_{1}, x_{2}, \ldots\right\}$, и $A$ - подпространство в данной алгебре (множество элементов в группоиде). Для любого набора $Y=\left\{x_{i_{1}}, \ldots, x_{i_{n}}\right\} \subset$ $X$ различных элементов обозначим через $P_{n}(A, Y)$ линейную оболочку (множество) всех полилинейных элементов степени $n$ от $Y$ в $A$. Пусть размерность этой линейной оболочки (число элементов) $c_{n}(A, Y)$ не зависит от выбора $Y$, а зависит только от $n$. Тогда обозначим $c_{n}=c_{n}(A, Y)$ и будем говорить, что для $A$ определена функция сложности относительно множества $X$ :

$$
\mathscr{C}(A, z)=\sum_{n=1}^{\infty} \frac{c_{n}}{n !} z^{n}, \quad z \in \mathbb{C},
$$

(для ассоциативных алгебр и группоидов с единицей сумма берется с $n=0$, при этом $\left.c_{0}=1\right)$.

Лемма 2 [6]. Пусть Г - группоид (алгебра), порожденный счетным множеством $X$. Предположим, что для подмножеств (подпространств) $A \subset \Gamma, B \subset \Gamma$ определены функции сложности.

1. Пусть $U=A \cup B, A \cap B=\varnothing,(U=A \oplus B-$ прямая сумма $)$. Тогда $U$ обладает функиией сложности, причем $\mathscr{C}(U, z)=\mathscr{C}(A, z)+\mathscr{C}(B, z)$.

2. Пусть все элементы $a * b, a \in A, b \in B$, множества $A * B \subset \Gamma$ различны (линейно независимы), где “*”- произведение в группоиде (алгебре). Тогда $A * B$ обладает функиией сложности, причем $\mathscr{C}(A * B, z)=\mathscr{C}(A, z) \mathscr{C}(B, z)$.

Пусть $f(n)$ и $g(n)$ - две функции натурального аргумента. Будем обозначать $f(n) \approx$ $g(n)$, если $\lim _{n \rightarrow \infty} f(n) / g(n)=1$.

Теорема 2. Для многообразия алгебр Лейбница $\widetilde{\mathbf{N}_{s} \mathbf{A}}$ над произвольным полем вернь следующие утверждения:

1) $\widetilde{\mathbf{N}_{s} \mathbf{A}}$ имеет следуюшую функиию сложности:

$$
\mathscr{C}\left(\widetilde{\mathbf{N}_{s} \mathbf{A}}, z\right)=z+\frac{z^{2}}{z-1}\left((1+(z-1) \exp (z))^{s}-1\right)
$$

2) для любого $n \geqslant 2$ выполнено равенство

$$
c_{n}\left(\widetilde{\mathbf{N}_{s} \mathbf{A}}\right)=\sum_{k=1}^{s} k^{n} \sum_{i=0}^{k-1} C_{s}^{k} C_{k-1}^{i}(-1)^{k-1-i} k^{-i-2} \frac{n !}{(n-i-2) !} ;
$$

3) $c_{n}\left(\widetilde{\mathbf{N}_{s} \mathbf{A}}\right) \approx n^{s+1} s^{n-s-1}, n \rightarrow \infty$.

ДоказАтельство. 1) Учитывая теорему 1 , пространство $P_{n}\left(\widetilde{\mathbf{N}_{s} \mathbf{A}}\right)$ представимо в виде прямой суммы $P_{n}\left(\widetilde{\mathbf{N}_{s} \mathbf{A}}\right) \cong \bigoplus_{c=0}^{s-1} R_{c, n}$, где $R_{c, n}$ является линейной оболочкой элементов 
вида (1) с условиями (2) и фиксированным значением $c$. Учитывая лемму 2, получаем

$$
\begin{aligned}
\mathscr{C}\left(R_{0, n}, z\right) & =\exp (z) z^{2}, \\
\mathscr{C}\left(R_{i, n}, z\right) & =\exp (z) z^{2}(z(\exp (z)-1)-(\exp (z)-1-z))^{i} \\
& =\exp (z) z^{2}(1+(z-1) \exp (z))^{i}, \quad i=1, \ldots, s-1, \\
\mathscr{C}\left(\widetilde{\mathbf{N}_{s} \mathbf{A}}, z\right) & =z+\exp (z) z^{2}\left(1+\sum_{i=1}^{s-1}(1+(z-1) \exp (z))^{i}\right) .
\end{aligned}
$$

Осталось привести функцию сложности $\mathscr{C}\left(\widetilde{\mathbf{N}_{s} \mathbf{A}}, z\right)$ к требуемому виду, учитывая равенство $C_{k}^{k}+C_{k+1}^{k}+\cdots+C_{n}^{k}=C_{n+1}^{k+1}$ (см. [9]).

2) Запишем функцию сложности в виде

$$
\mathscr{C}\left(\widetilde{\mathbf{N}_{s} \mathbf{A}}, z\right)=z+\sum_{k=1}^{s} C_{s}^{k} z^{2}(z-1)^{k-1} \exp (k z) .
$$

При этом

$$
\begin{aligned}
z^{2}(z-1)^{k-1} \exp (k z) & =\sum_{i=0}^{k-1} C_{k-1}^{i} z^{i+2}(-1)^{k-1-i} \sum_{m=0}^{\infty} \frac{k^{m}}{m !} z^{m} \\
& =\sum_{i=0}^{k-1} \sum_{m=0}^{\infty} C_{k-1}^{i}(-1)^{k-1-i} z^{m+i+2} \frac{k^{m}}{m !} \\
\stackrel{m+i+2}{=}=n & \sum_{i=0}^{k-1} \sum_{n=i+2}^{\infty} C_{k-1}^{i}(-1)^{k-1-i} \frac{k^{n-i-2}}{(n-i-2) !} z^{n} \\
& =\sum_{n=1}^{\infty}\left(\sum_{i=0}^{k-1} C_{k-1}^{i}(-1)^{k-1-i} \frac{1}{k^{i+2}} \frac{n !}{(n-i-2) !}\right) \frac{k^{n}}{n !} z^{n} .
\end{aligned}
$$

В последнем равенстве использовался тот факт, что $n ! /(n-i-2) !=0$ при $n-i-2<0$. Подставим последнюю двойную сумму в (3):

$$
z+\sum_{n=1}^{\infty} \frac{z^{n}}{n !}\left(\sum_{k=1}^{s} k^{n} \sum_{i=0}^{k-1} C_{s}^{k} C_{k-1}^{i}(-1)^{k-1-i} \frac{1}{k^{i+2}} \frac{n !}{(n-i-2) !}\right) .
$$

То, что находится внутри скобок, и есть $c_{n}\left(\widetilde{\mathbf{N}_{s} \mathbf{A}}\right), n \geqslant 2$.

3) Следует из пункта 2.

\section{СПИСОК ЦИТИРОВАННОЙ ЛИТЕРАТУРЫ}

[1] J.-L. Loday, Enseign. Math. (2), 39:3-4 (1993), 269-293. [2] Ю. А. Бахтурин, Тождества в алгебрах Ли, Наука, М., 1985. [3] С. М. Рацеев, Вестн. СамГУ. Естественнонаучн. сер., 2006, №6/1, 70-77. [4] С. М. Рацеев, Матем. заметки, 82:1 (2007), 108-117. [5] С. М. Рацеев, Вестн. СамГУ. Естественнонаучн. сер., 2010, № 4, 65-72. [6] Я. Гульден, Д. Джексон, Перечислительная комбинаторика, Наука, М., 1990. [7] В. М. Петроградский, Фундамент. и прикл. матем., 1:4 (1995), 989-1007. [8] В. М. Петроградский, Матем. сб., 188:6 (1997), 119-138. [9] Р. Грэхем, Д. Кнут, О. Паташник, Конкретная математика. Основание информатики, Мир, М., 1998.

С. М. Рацеев

Ульяновский государственный университет

E-mail: RatseevSM@mail.ru
Поступило

23.12 .2014

Исправленный вариант 